\title{
Longitudinal Changes in Myocardial T-1 and T-2 Relaxation Times Related to Diffuse Myocardial Fibrosis in Aortic Stenosis; Before and After Aortic Valve Replacement
}

Sofia Kvernby, Mattias Rönnerfalk, Marcel J an Bertus Warntjes, Carljohan Carlhäll, Eva Nylander, J an Engvall, Eva Tamas and Tino Ebbers

The self-archived postprint version of this journal article is available at Linköping University Institutional Repository (DiVA):

http:/ / urn.kb.se/ resolve?urn=urn:nbn:se:liu:diva-151493

N.B.: When citing this work, cite the original publication.

Kvernby, S., Rönnerfalk, M., Warntjes, M. J . B., Carlhäll, C., Nylander, E., Engvall, J., Tamas, E., Ebbers, T., (2018), Longitudinal Changes in Myocardial T-1 and T-2 Relaxation Times Related to Diffuse Myocardial Fibrosis in Aortic Stenosis; Before and After Aortic Valve Replacement, J ournal of Magnetic Resonance Imaging, 48(3), 799-807. https:// doi.org/ 10.1002/jmri.25980

Original publication available at:

https:/ / doi.org/ 10.1002/jmri.25980

Copyright: Wiley

http:/ / eu.wiley.com/WileyCDA/ 


\section{Longitudinal changes in myocardial $\mathrm{T} 1$ and $\mathrm{T} 2$ relaxation times related to diffuse}

myocardial fibrosis in aortic stenosis; before and after aortic valve replacement

Kvernby Sofia, M.Sc ${ }^{1,2}$, Rönnerfalk Mattias, M.D ${ }^{3}$, Warntjes Marcel, Ph.D ${ }^{2,4,5}$, Carlhäll Carl-Johan, M.D, Ph.D ${ }^{2,6}$, Nylander Eva, M.D, Ph.D ${ }^{6}$, Engvall Jan, M.D, Ph.D ${ }^{2,6}$, Tamás Éva, M.D, Ph.D ${ }^{2,3}$ and Ebbers Tino, Ph.D $\mathrm{D}^{2,4} \S$

${ }^{1}$ Department of Radiation Physics and Department of Medical and Health Sciences, Linköping University, Linköping, Sweden

${ }^{2}$ Center for Medical Image Science and Visualization (CMIV), Linköping University, Sweden

${ }^{3}$ Department of Cardiothoracic and Vascular Surgery and Department of Medical and Health Sciences, Linköping University, Linköping, Sweden

${ }^{4}$ Department of Medical and Health Sciences, Linköping University, Linköping, Sweden ${ }^{5}$ SyntheticMR AB, Linköping, Sweden

${ }^{6}$ Department of Clinical Physiology and Department of Medical and Health Sciences, Linköping University, Linköping, Sweden

$\S^{\S}$ Corresponding author

Email addresses: SK: sofia.kvernby@liu.se

MR: mattias.ronnerfalk@regionostergotland.se

MW: marcel.jan.bertus.warntjes@liu.se

CJC: carljohan.carlhall@liu.se

EN: eva.nylander@regionostergotland.se

JE: jan.engvall@regionostergotland.se

ET: eva.tamas@regionostergotland.se

TE: tino.ebbers@liu.se, +46 10-103 33 11, Linköping University, Institutionen för medicin och hälsa, 58183 Linköping, Sweden 


\section{Acknowledgements}

We acknowledge statistician Mats Fredriksson for providing valuable advice related to the statistical methods used in this study.

\section{Grant support}

This work was partly supported by grants from the Swedish Research Council, the Swedish Heart and Lung foundation, the Swedish Society of Medicine and ALF Grants Region Östergötland.

Running title

Myocardial relaxation times after AVR 
1 Longitudinal changes in myocardial $\mathrm{T} 1$ and $\mathrm{T} 2$ relaxation times related to diffuse

2 myocardial fibrosis in aortic stenosis; before and after aortic valve replacement

3

\section{Abstract}

5 Background

6 Diffuse myocardial fibrosis is connected to adverse outcome in several cardiac diseases, but

7 detection and quantification is challenging. Cardiovascular magnetic resonance (MR)

8 relaxation times mapping represent promising imaging biomarkers for diffuse myocardial

9 fibrosis.

10 Purpose/Hypothesis

11 The objective of this study was to investigate whether myocardial relaxation times can detect

12 longitudinal changes in myocardial tissue composition associated with diffuse fibrosis in

13 patients with severe aortic stenosis (AS) before and after aortic valve replacement (AVR).

14 Study type

15 Prospective longitudinal study.

16 Population/Subjects/Phantom/Specimen/Animal model

17 Fifteen patients with severe AS.

18 Field strength/Sequence

193 Tesla / 3(3)3(3)5-MOLLI, T2-GraSE and 3D-QALAS

20 Assessment

21 Fifteen patients with severe AS accepted for AVR underwent MR examinations at three time

22 points: before AVR, as well as 3 and 12 months after AVR. The MR examinations included a

23 multiple-slice cine acquisition, 2D-MOLLI acquisitions for T1-mapping, 2D-GraSE

24 acquisitions for T2-mapping and a 3D-QALAS acquisition for combined T1- and T2-

25 mapping. Data from each patient were analyzed in 16 myocardial segments. 


\section{Statistical tests}

2 The segment-wise T1 and T2 data were analyzed over time after surgery using linear mixed

3 models with repeated measures.

\section{$4 \quad$ Results}

5 The results showed that T1 relaxation times were significantly $(\mathrm{p}<0.05)$ shorter 3 and 12

6 months postoperative than pre-operative and that the T2 relaxation times were significantly

$7 \quad(\mathrm{p}<0.05)$ longer 3 and 12 months postoperative than pre-operative for both 3D- and 2D-

8 mapping methods. No significant changes were seen between 3 and 12 months postoperative.

\section{Data conclusion}

10 Our findings demonstrate that changes in myocardial relaxation times and thus tissue

11 characteristics can be observed already early after AVR surgery, within the first 3 months.

12 The significant changes in relaxation times from pre-operative examinations to the follow up 13 may be interpreted as a reduction of interstitial fibrosis in the left ventricular wall.

Keywords

17 Relaxation times mapping, Aortic valve replacement, Longitudinal study, Aortic stenosis, 18 Remodeling, Fibrosis 


\section{Introduction}

2 Diffuse fibrosis is connected to an adverse outcome in several cardiac diseases including

3 aortic stenosis (AS), but detection and quantification has shown to be challenging(1). In AS

4 the left ventricular outflow is obstructed, which results in a pressure gradient across the aortic

5 valve. In patients with severe AS, this often leads to concentric left ventricular hypertrophy to

6 maintain adequate cardiac output. Left ventricular hypertrophy due to chronic pressure

7 overload is associated with normal intra-cavity size, but increased wall thickness. An

8 increased amount of interstitial fibrosis is present in concentric hypertrophy due to AS (2).

9 The increased amount of interstitial fibrosis is assumed to be diffusely distributed in the

10 myocardium. Myocardial fibrosis may be reversible(3), but among AS patients, presence of

11 myocardial fibrosis has shown to dramatically increase the all-cause mortality risk $(4,5)$, even

12 after aortic valve replacement(6). The possibility to detect and quantify diffuse myocardial

13 fibrosis at an early stage of the disease is therefore of clinical importance, both to quantify

14 myocardial disease burden and to optimize timing of intervention.

Non-invasive methods to quantify diffuse myocardial fibrosis are lacking. Myocardial biopsies allow for analysis of myocardial fibrosis, but this approach is invasive and provides only regional information. Cardiovascular magnetic resonance allows, by the use of delayed contrast enhancement, for detection of focal myocardial fibrosis, but falls short in detection of diffuse fibrosis since normal unaffected myocardium is missing. Quantitative MR imaging and MR relaxation times mapping have shown to represent promising imaging biomarkers for diffuse myocardial fibrosis(7-9), but longitudinal studies using this methods are missing.

24 In the field of myocardial relaxation times mapping in relation to diffuse fibrosis, focus has mostly been on the longitudinal T1 relaxation. The longitudinal spin-lattice relaxation time, 
1 T1, can be described as a loss of energy among the spins and as the recovery of the

2 magnetization vector in $\mathrm{z}$-direction, i.e. parallel to the static magnetic field. The relaxation is

3 caused by dipole-dipole interactions(10), which are induced by fluctuations in the local

4 magnetic field. Thermal molecular motions, such as rotations and vibrations, cause the

5 fluctuations in the local magnetic field. Composition and solidity of a tissue determine how

6 fast, and with what frequency, a dipole-dipole interaction can occur and thus how fast the T1

7 relaxation is. The fastest, i.e. shortest $\mathrm{T} 1$ relaxation time is found in tissue where fluctuations

8 in the local magnetic field occur with a frequency close to the Larmor frequency(11). Studies

9 have demonstrated a correlation between diffuse myocardial fibrosis and long native T1

10 relaxation time $(12,13)$. A short $\mathrm{T} 1$ relaxation time in contrast enhanced myocardial tissue has

11 also been correlated to increased amount of diffuse fibrosis(14-16).

13 The relationship between the transverse relaxation time, T2, and myocardial fibrosis is not

14 studied to the same extent. The transverse spin-spin relaxation time can be described as loss

15 of phase coherence among the spins in the transverse plane, resulting in an overall loss of

16 signal. The loss of phase coherence is mostly due to magnetic interactions and magnetic

17 disturbances with the surrounding molecules. How fast the spins tumble through space is a

18 key aspect in the transverse relaxation. The faster they tumble, the longer the transverse

19 relaxation time becomes. Larger water content enables spins to tumble faster and imply longer

20 T2 relaxation time. In animal studies, correlation between myocardial fibrosis and T2

21 relaxation time has shown contradictory results. In mice studies, myocardial fibrosis has been

22 correlated to both prolonged T2 relaxation time (17) and shortened T2 relaxation time

23 (18,19), but there is a lack of studies in human myocardial tissue. 
1 The aim of this study was to investigate whether myocardial T1 and T2 relaxation times can

2 detect longitudinal changes in myocardial tissue composition associated with diffuse

3 myocardial fibrosis in patients with severe AS prior to, three and 12 months after aortic valve 4 replacement (AVR).

\section{Materials and methods}

7 All subjects gave written informed consent to participate in the study and approval was 8 granted from the Regional Ethical Review Board.

\section{Study population and MR examination}

11 This study included patients with high gradient severe AS accepted for AVR between April 2014 and December 2015. Patients were accepted for surgical intervention according to current guidelines(20). Patients with a history of any other concomitant cardiac disease, or suffering from arrhythmias or claustrophobia were excluded. Fifteen patients (8 male, 7 female) accepted to participate in the study (Table 1).

The patients underwent MR examinations at three different time points: before AVR, as well as 3 and 12 months after AVR. The MR examinations included a multiple-slice short-axis cine balanced steady-state free precession (SSFP) acquisition, native 2D MOLLI (21) T1 acquisitions and 2D T2-GraSE acquisitions, at apical, mid-ventricular and basal slice

21 positions. The patients also underwent a native 3D-QALAS (22) acquisition. All scans were performed using a Philips Ingenia 3T system.

24 The MOLLI acquisitions were performed using 3(3)3(3)5 acquisitions. The resolution was

$251.22 \mathrm{~mm} \times 1.22 \mathrm{~mm}$ in plane and with a slice thickness of $10 \mathrm{~mm}$. A flip angle of $35^{\circ}$ was 
1 used together with a repetition time of $2.4 \mathrm{~ms}$ and an echo time of $1.13 \mathrm{~ms}$. The MOLLI

2 acquisition was performed during a breath hold of 17 heartbeats and provided one 2D slice of

3 the left ventricular myocardium.

4

5 The T2-GraSE acquisitions, also providing one 2D slice, had the same resolution as the

6 MOLLI acquisitions and were performed during a breath hold of 15 heartbeats. Nine different

7 echo times, range between $9 \mathrm{~ms}$ and $84 \mathrm{~ms}$, were used for the calculation of the T2-map and

8 the repetition time was $1000 \mathrm{~ms}$.

9

The 3D-QALAS method provides full 3D coverage of the LV myocardium with simultaneous mapping of T1- and T2 relaxation times in one breath hold. The 3D-QALAS acquisition had a resolution of $2.0 \mathrm{~mm} \times 2.0 \mathrm{~mm}$ in-plane and a slice thickness of $12.0 \mathrm{~mm}$ (reconstructed to $2.0 \mathrm{~mm} \times 2.0 \mathrm{~mm} \times 6.0 \mathrm{~mm}$ ), flip angle of $5^{\circ}$, SENSE factor of 2 in phase direction and $1.2 \mathrm{in}$ slice direction, repetition time was $2.6 \mathrm{~ms}$ and echo time was $1.2 \mathrm{~ms}$. The 3D-QALAS acquisition was performed during a breath hold of 15 heartbeats and provided 13 short-axis slices of the left ventricular myocardium.

\section{Image analysis}

Quantitative images from MOLLI and GraSE were generated immediately on the scanner console. The 3D-QALAS data were exported from the scanner console and quantitative images, i.e. T1- and T2 maps, were generated in a standalone version of SyMRI ${ }^{\circledR}$ (SyntheticMR, Sweden). Analysis of the generated relaxation times maps were performed in Segment v 1.9 R3644 (http://segment.heiberg.se). Epi- and endocardial borders were manually contoured in the thirteen (representing the thirteen slices of the left ventricular myocardium) T1- and T2 maps of each 3D-QALAS acquisition and in the three T1- and T2 
1 maps from the 2D methods. Based on these manual contours, 16-segment bull's eye plots

2 were created automatically by the analysis software, excluding the apical cap that is not

3 accessible from the short-axis acquisitions. The 16 segments were in accordance with the

4 myocardial segment model recommended by the American Heart Association (AHA)(23). For

5 each time point and each patient, an average value for T1- and T2 relaxation time were given

6 for the area included in each segment of the 16-segment bull's eye plot.

7

8 The balanced cine SSFP MR images were used to measure left ventricular wall mass,

9 volumes and ejection fraction at the three different time points using a later version of

10 Segment, v.2.1 R5726.

11

12 Statistical analysis

13 Segmental myocardial T1- and T2 relaxation times were compared between the three different

14 time points. Data analysis was performed with IBM SPSS Statistics, Version 24.0 (Armonk,

15 NY: IBM Corp.). All data were normally distributed according to the Kolmogorov-Smirnov

16 test, thus parametric tests were used for the statistical analysis. In order to investigate

17 differences over time, linear mixed models with repeated measures were used for segment-

18 wise data. The different time points were defined as a fixed effect and the patients were

19 defined as a random effect. Estimates of fixed effects will provide information about

20 significant changes in relaxation times over the three time points. Statistical significance was

21 set as $\mathrm{p}<0.05$.

22

23 Results

24 Data were successfully acquired in all cases. 
1 Patient characteristics

2 Heart rate, blood pressure and left ventricular ejection fraction were unaltered pre- versus

3 postoperatively. A significant reduction in left ventricular wall mass $(\mathrm{p}<0.005)$ was seen from

4 pre- to postoperatively. Characteristics of the patients, together with clinical parameters, can

5 be seen in Table 1.

6

$7 \quad$ Cardiovascular magnetic resonance $\mathrm{T} 1$ and $\mathrm{T} 2$ relaxation times

8 To investigate changes in myocardial relaxation times longitudinally, the same myocardial

9 segment from the same patient was followed over time. For both methods, the results showed

10 that the T1 relaxation times were significantly shorter 3 and 12 months follow-up than pre-

11 operatively (Figure 1) and that the T2 relaxation times were significantly longer 3 and 12

12 months follow-up than pre-operatively (Figure 2). No significant difference was seen in T1 or

13 T2 relaxation times between 3 months and 12 months follow-up. A summary of the results is

14 presented in Table 2.

15

16 For the 3D-method (3D-QALAS), the overall average native T1/T2 value from the 16

17 segments in all patients was $1169 \mathrm{~ms} / 48 \mathrm{~ms}$ pre-operatively, $1142 \mathrm{~ms} / 50 \mathrm{~ms}$ at the 3 months

18 follow-up and $1126 \mathrm{~ms} / 51 \mathrm{~ms}$ at the 12 months follow up (3.7\% decrease in $\mathrm{T} 1$ and $4.6 \%$

19 increase in T2 values). For the 2D-methods (MOLLI and GraSE), the overall average T1/T2

20 value from the 16 segments in all patients was $1187 \mathrm{~ms} / 50 \mathrm{~ms}$ pre-operatively, $1165 \mathrm{~ms} / 52$

$21 \mathrm{~ms}$ at the 3 months follow-up and $1160 \mathrm{~ms} / 53 \mathrm{~ms}$ at the 12 months follow up (2.3\% decrease

22 in $\mathrm{T} 1$ and $4.3 \%$ increase in $\mathrm{T} 2$ ).

23

24 In figure 3, the average percentage difference between follow up and preoperatively, are

25 presented in a segment-wise manner and color-coded to get an overview of the myocardial 
1 relaxation times data. Figure 4 shows mid-ventricular T1- and T2-maps acquired with 3D-

2 QALAS, MOLLI and T2-GraSE at the three different time points in one of the patients.

\section{Discussion}

5 In this study, we show that myocardial relaxation times, both T1 and T2 display changes in

6 patients with severe AS from before AVR to 12 months after AVR that are statistically

7 significant. Native myocardial T1 relaxation time was found to significantly decrease from

8 before surgery to 12 months after surgery and myocardial T2 relaxation time was found to

9 significantly increase from before surgery to 12 months after surgery. A significant decrease

10 in T1 and increase in T2 could already be seen at the 3 months follow up. Furthermore, there

11 was in general a good consistency in the results between the 2D-mapping methods and the 12 3D-mapping method used in this study.

14 Comparing these findings with T1 and T2 values in healthy volunteers is not obvious, as the

normal range of relaxation times is large in a healthy cohort and even larger in cohorts of patients with myocardial pathologies. If such a comparison is made anyway, it is important to compare values acquired with the same mapping sequence and from the same specific site, as recommended by the T1 mapping consensus statement(24) and a T1 mapping review article by Puntmann et al (25). Previously, T1 and T2 relaxation times of healthy cohorts have been published from this site using the same mapping sequences as in this study $(22,26)$. Pooling the 20 healthy volunteers from these two studies, the average T1 relaxation times were 1131 ms and 1117 ms for 3D-QALAS respectively MOLLI, and the average T2 relaxation times were $51.6 \mathrm{~ms}$ and $49.2 \mathrm{~ms}$ for 3D-QALAS respectively T2-GraSE. For all relaxation times we see the same trend over time after surgery, that the relaxation times for the aortic stenosis patients approach those of the healthy cohort, except for T2-GraSE. However, in this study 
1 our main focus is not on differences between patients and healthy subjects. Instead, we have

2 chosen to follow the same patient over time and thus focus on changes in myocardial

3 relaxation times on a longitudinal basis.

4

5 Interstitial fibrosis in AS patients has in several studies been associated with increased native

6 myocardial T1 relaxation time $(12,13,27)$ but, to our knowledge, the association with the

7 effect of reversed remodeling after surgical intervention has not been studied. Our findings,

8 which focus on individual changes in relaxation times after AVR, demonstrate a decrease in

9 native T1 relaxation time from before AVR to 12 months after AVR, which may be

10 interpreted as a reduction of interstitial fibrosis. The largest decrease in T1 relaxation time

11 occurs between pre AVR and 3 months post AVR, for both the MOLLI method and the 3D-

12 QALAS method (Figure 1). Between 3 months and 12 months of follow up, only a slight non13 significant additional decrease in T1 can be noted.

The transverse T2 relaxation time was also investigated with respect to changes over time after surgery. For both methods, a significant increase in T2 times was found at the 12 months follow up, compared with pre-operatively. Shorter T2 relaxation time before surgery and longer T2 relaxation time after surgery may indicate an increased tumbling speed of spins, following the Bloembergen-Purcell-Pound (BPP) theory (28), and thus increased relative amount of water content in the tissue after surgery. Assuming that the included patients have elevated amounts of interstitial fibrosis before the AVR, fibrotic myocardial tissue is thus stiffer with lower water content and spins tumble slower through space, implying more interactions with surrounding molecules and thereby shorter T2 relaxation time. However, contradicting results have been shown in animal studies, correlating collagen level or the amount of fibrotic tissue with both longer and shorter T2 relaxation times (17-19). The 
1 association between myocardial interstitial fibrosis and T2 relaxation time in human myocardial tissue found in this study may provide important addition to our body of knowledge of myocardial tissue composition in the setting of fibrosis.

Myocardial edema, myocardial inflammation and iron content are also known to affect MR relaxation times, and can therefore potentially also contribute to the changes found in this study. Especially myocardial edema and inflammation related to surgery are possible confounders of changes in the MR relaxation times. However, a potential presence of myocardial edema or inflammation after surgery is expected to result in an increase in T1 as well as T2 after surgery(29), while we observed an increase in T2 in combination with a decrease in T1. Also the fact that T1 and T2 after surgery change towards normal values indicates that the amount of edema or inflammation is relatively small 3 and 12 months after surgery, which is in accordance with Dongaonkar et al(30). The observed decrease in left ventricular mass after surgery may also affect the relaxation times. A left ventricular mass reduction solely caused by a reduction of muscle tissue, would result in a relative increase in fibrotic tissue and consequently an increased T1. We observed a decreased T1, which indicates a larger reduction of fibrotic tissue than muscle tissue. The results therefore seem to be predominantly explained by a reduction of myocardial fibrosis over time after AVR.

According to studies based on endomyocardial biopsies (31) and late gadolinium enhancement (LGE) MR (32), fibrotic tissue and left ventricular hypertrophy often decreases after AVR. The decrease in left ventricular hypertrophy was in those studies found at an early stage, while the decrease in fibrotic tissue could be seen first after a longer period of time. In this study, changes in myocardial relaxation times, together with left ventricular mass, are seen already at an early stage (three months) after AVR. In contrast to data based on LGE and 
1 biopsies, our findings propose that myocardial relaxation times, both T1 and T2, are early

2 markers of changes in myocardial tissue characteristics and may reflect the amount of fibrotic 3 tissue after AVR.

4

5 For each subject, myocardial relaxation times have been analyzed on a segment-wise manner

6 over time. Differences between pre-operatively and follow up were calculated for each

7 segment and are illustrated in figure 3 . The figure demonstrates variations of the differences

8 in relaxation times over the myocardial segments. In general, the variations between the

9 segments seem to be a little larger for the 2D-methods than for the 3D-method. One factor

10 may be the use of an iterative analysis approach instead of a conventional curve fitting

11 approach for the 3D-QALAS method. This iterative approach handles several possibly

12 confounding factors that could affect the estimate of T1 and T2, and makes the method more

13 stable. The difference between the 2D methods and the 3D method may also be explained by

14 issues arising in scan - rescan situations, such as positioning of the shim-box, the exact slice

15 positioning and inflow effects, that seem to influence the 2D-methods more than the 3D-

16 method (26). Comparing preoperative data with the 12 months follow up (right column in

17 figure 3), there seems to be a larger change in relaxation times in the septal segments than in

18 the lateral segments of the left ventricular myocardium. This observation is not statistically

19 significant but is thought provoking and may indicate that septal segments are more likely to

20 undergo a larger positive remodeling with respect to hypertrophic response than for example

21 lateral segments, in accordance with Dweck et al (33).

22

23 Recently, studies have shown an inability for relaxation times mapping methods to separate

24 healthy myocardium from pathological myocardium on an individual basis, due to the broad

25 range of normal tissue relaxation times values(34,35). In our opinion, the strength and the 
1 clinical potential of relaxation times mapping lies in longitudinal studies, i.e. following the

2 same subject over time. In such studies, the naturally occurring differences in relaxation times

3 between subjects are not of importance. So far, there is a lack of studies illustrating the

4 potential of using myocardial relaxation times in longitudinal studies, as well as using the

5 combination of T1- and T2 relaxation times in patients with severe AS. This study therefore

6 contributes with new applications in the field, combined myocardial T1- and T2 mapping in a

7 longitudinal manner.

8

\section{Limitations}

10 This study contains a limited number of included patients. However, despite the low number

11 of patients, we found significant changes in MR relaxation times over time after AVR.

12 Myocardial relaxation times representing normal tissue are well known to differ depending on

13 for example vendor and mapping method used (36), which is a limitation when generalizing

14 values from a single center. According to that, we have chosen to include both $3 \mathrm{D}$ and 2D

15 mapping methods in this study, which we see as a strength, as all methods show significant

16 results.

\section{Conclusions}

19 Our findings demonstrate that changes in myocardial relaxation times and thus tissue

20 characteristics can be observed already early after AVR surgery, within the first 3 months.

21 The significant changes in relaxation times from pre-operative examinations to the follow up

22 may be interpreted as a reduction of interstitial fibrosis in the left ventricular wall. 


\section{References}

2

3 1. Neubauer S, Bull S. Myocardial Fibrosis in Aortic Stenosis. JACC: Cardiovascular $4 \quad$ Imaging 2016.

5 2. Mann DL, Zipes DP, Libby P, Bonow RO. Braunwald's heart disease: a textbook of cardiovascular medicine: Elsevier Health Sciences: 2014.

3. Krayenbuehl HP, Hess OM, Monrad ES, Schneider J, Mall G, Turina M. Left ventricular myocardial structure in aortic valve disease before, intermediate, and late after aortic valve replacement. Circulation 1989;79(4):744-755.

4. Dweck MR, Joshi S, Murigu T, et al. Midwall Fibrosis Is an Independent Predictor of Mortality in Patients With Aortic Stenosis. Journal of the American College of

5. O'Hanlon R, Grasso A, Roughton M, et al. Prognostic significance of myocardial fibrosis in hypertrophic cardiomyopathy. J Am Coll Cardiol 2010;56(11):867-874.

6. Azevedo CF, Nigri M, Higuchi ML, et al. Prognostic Significance of Myocardial Fibrosis Quantification by Histopathology and Magnetic Resonance Imaging in Patients With Severe Aortic Valve Disease. Journal of the American College of Cardiology 2010;56(4):278-287.

7. Mewton N, Liu CY, Croisille P, Bluemke D, Lima JA. Assessment of myocardial fibrosis with cardiovascular magnetic resonance. J Am Coll Cardiol 2011;57(8):891-903.

8. Bulluck H, Maestrini V, Rosmini S, et al. Myocardial T1 Mapping- Hope or Hype? Circulation Journal 2015;79(3):487-494. 
1 9. White SK, Sado DM, Flett AS, Moon JC. Characterising the myocardial interstitial space: the clinical relevance of non-invasive imaging. Heart 2012;98(10):773779.

10. Levitt MH. Spin dynamics: basics of nuclear magnetic resonance: John Wiley \& Sons: 2001 p: 533.

11. Reimer P, Parizel PM. Clinical MR imaging: Springer: 2010 p: 3-5.

12. Dass S, Suttie JJ, Piechnik SK, et al. Myocardial Tissue Characterization Using Magnetic Resonance Noncontrast T1 Mapping in Hypertrophic and Dilated Cardiomyopathy. Circulation: Cardiovascular Imaging 2012;5(6):726-733.

13. Bull S, White SK, Piechnik SK, et al. Human non-contrast T1 values and correlation with histology in diffuse fibrosis. Heart 2013.

14. Iles L, Pfluger H, Phrommintikul A, et al. Evaluation of Diffuse Myocardial Fibrosis in Heart Failure With Cardiac Magnetic Resonance Contrast-Enhanced T1 Mapping. Journal of the American College of Cardiology 2008;52(19):1574-1580.

15. Flett AS, Hayward MP, Ashworth MT, et al. Equilibrium contrast cardiovascular magnetic resonance for the measurement of diffuse myocardial fibrosis

16. Ellims AH, Iles LM, Ling LH, Hare JL, Kaye DM, Taylor AJ. Diffuse myocardial

17. Grover-McKay M, Scholz TD, Burns TL, Skorton DJ. Myocardial collagen Cardiovasc Magn Reson 2012;14:76. concentration and nuclear magnetic resonance relaxation times in the spontaneously hypertensive rat. Invest Radiol 1991;26(3):227-232. 
1 18. Bun S-S, Kober F, Jacquier A, et al. Value of In Vivo T2 Measurement for Myocardial Fibrosis Assessment in Diabetic Mice at 11.75 T. Investigative Radiology 2012;47(5):319-323.

19. Loganathan R, Bilgen M, Al-Hafez B, Smirnova IV. Characterization of Alterations in Diabetic Myocardial Tissue Using High Resolution MRI. The International Journal of Cardiovascular Imaging 2006;22(1):81-90.

20. Nishimura RA, Otto CM, Bonow RO, et al. 2014 AHA/ACC guideline for the management of patients with valvular heart disease. Circulation 2014:CIR. 0000000000000031.

21. Messroghli DR, Radjenovic A, Kozerke S, Higgins DM, Sivananthan MU, Ridgway JP. Modified Look-Locker inversion recovery (MOLLI) for high-resolution T1 mapping of the heart. Magn Reson Med 2004;52.

22. Kvernby S, Warntjes MJB, Haraldsson H, Carlhäll C-J, Engvall J, Ebbers T. Simultaneous three-dimensional myocardial T1 and T2 mapping in one breath hold with 3D-QALAS. Journal of Cardiovascular Magnetic Resonance 2014;16(1):1-14.

23. Cerqueira MD, Weissman NJ, Dilsizian V, et al. Standardized myocardial segmentation and nomenclature for tomographic imaging of the heart a statement for healthcare professionals from the cardiac imaging committee of the Council on Clinical Cardiology of the American Heart Association. Circulation 2002;105(4):539-542.

24. Moon JC, Messroghli DR, Kellman P, et al. Myocardial T1 mapping and extracellular volume quantification: a Society for Cardiovascular Magnetic Resonance (SCMR) and CMR Working Group of the European Society of Cardiology consensus statement. J Cardiovasc Magn Reson 2013;15(1):92. 
1 25. Puntmann V0, Peker E, Chandrashekhar Y, Nagel E. T1 Mapping in Characterizing Myocardial Disease. A Comprehensive Review 2016;119(2):277-299.

26. Kvernby S, Warntjes M, Engvall J, Carlhäll C-J, Ebbers T. Clinical feasibility of 3DQALAS - Single breath-hold 3D myocardial T1- and T2-mapping. Magnetic Resonance Imaging 2017;38:13-20.

27. Lee S-P, Lee W, Lee JM, et al. Assessment of Diffuse Myocardial Fibrosis by Using MR Imaging in Asymptomatic Patients with Aortic Stenosis. Radiology 2015;274(2):359-369.

28. Bloembergen N, Purcell EM, Pound RV. Relaxation Effects in Nuclear Magnetic Resonance Absorption. Physical Review 1948;73(7):679-712.

29. Ugander M, Bagi PS, Oki AJ, et al. Myocardial Edema as Detected by Pre-Contrast T1 and T2 CMR Delineates Area at Risk Associated With Acute Myocardial Infarction. JACC: Cardiovascular Imaging 2012;5(6):596-603.

30. Dongaonkar RM, Stewart RH, Geissler HJ, Laine GA. Myocardial microvascular permeability, interstitial oedema, and compromised cardiac function. Cardiovascular research 2010;87(2):331-339.

31. Villari B, Vassalli G, Monrad ES, Chiariello M, Turina M, Hess OM. Normalization of Diastolic Dysfunction in Aortic Stenosis Late After Valve Replacement. Circulation 1995;91(9):2353-2358.

32. Weidemann F, Herrmann S, Störk S, et al. Impact of Myocardial Fibrosis in Patients With Symptomatic Severe Aortic Stenosis. Circulation 2009;120(7):577584.

33. Dweck MR, Joshi S, Murigu T, et al. Left ventricular remodeling and hypertrophy in patients with aortic stenosis: insights from cardiovascular magnetic resonance. Journal of Cardiovascular Magnetic Resonance 2012;14(1):50. 
1 34. von Knobelsdorff-Brenkenhoff F, Prothmann M, Dieringer MA, et al. Current T1 and T2 mapping techniques applied with simple thresholds cannot discriminate acute from chronic myocadial infarction on an individual patient basis: a pilot study. BMC Medical Imaging 2016;16(1):35.

35. Goebel J, Seifert I, Nensa F, et al. Can Native T1 Mapping Differentiate between Healthy and Diffuse Diseased Myocardium in Clinical Routine Cardiac MR Imaging? PLoS ONE 2016;11(5):e0155591.

36. Kellman P, Hansen MS. T1-mapping in the heart: accuracy and precision. Journal of Cardiovascular Magnetic Resonance 2014;16(1):2.

1 


\section{Tables}

2

3 Table 1. Patient characteristics presented as mean \pm one standard deviation.

\begin{tabular}{|l|l|l|l|}
\hline Parameter; average \pm sd & Before AVR & 3 months after & 12 months after \\
\hline Age [years] & $69 \pm 6$ & AVR \\
\hline Gender & 8 Male, 7 & & \\
\hline Systolic blood pressure [mmHg] & $142 \pm 12$ & $141 \pm 13$ & $143 \pm 9$ \\
\hline Diastolic blood pressure [mmHg] & $78 \pm 9$ & $83 \pm 8$ & $83 \pm 6$ \\
\hline Heart rate [bpm] & $63 \pm 9$ & $65 \pm 9$ & $65 \pm 10$ \\
\hline Left ventricular wall mass [g] & $152 \pm 38$ & $127 \pm 33$ & $117 \pm 28$ \\
\hline Left ventricular ejection fraction & $63 \pm 11$ & $63 \pm 6.1$ & $66 \pm 5.8$ \\
[\%] & \multicolumn{2}{|l}{} \\
\hline 4
\end{tabular}

5

6

7

8

9

10

11

12 
1 Table 2. Comparison of myocardial relaxation times by linear mixed model with repeated measures.

Linear mixed model (estimates and p-value of fixed effects)

\begin{tabular}{|c|c|c|c|}
\hline & $\begin{array}{l}\text { Pre-operatively vs } \\
\text { 3 months }\end{array}$ & $\begin{array}{l}\text { Pre-operatively vs } \\
12 \text { months }\end{array}$ & $\begin{array}{l}3 \text { months vs } \\
12 \text { months }\end{array}$ \\
\hline Native T1 3D- & Estimate: -40.2 & Estimate: -56.0 & Estimate: -15.9 \\
\hline QALAS & p-value $<0.05$ & p-value $<0.05$ & p-value: not sig. \\
\hline Native T1 MOLLI & $\begin{array}{l}\text { Estimate: }-18.4 \\
\text { p-value }<\mathbf{0 . 0 5}\end{array}$ & $\begin{array}{l}\text { Estimate: }-24.3 \\
\text { p-value }<\mathbf{0 . 0 5}\end{array}$ & $\begin{array}{l}\text { Estimate: }-5.93 \\
\text { p-value: not sig. }\end{array}$ \\
\hline T2 3D-QALAS & $\begin{array}{l}\text { Estimate: } 1.58 \\
\text { p-value }<\mathbf{0 . 0 5}\end{array}$ & $\begin{array}{l}\text { Estimate: } 2.26 \\
\text { p-value }<\mathbf{0 . 0 5}\end{array}$ & $\begin{array}{l}\text { Estimate: } 0.677 \\
\text { p-value: not sig. }\end{array}$ \\
\hline T2 GraSE & $\begin{array}{l}\text { Estimate: } 1.63 \\
\text { p-value }<\mathbf{0 . 0 5}\end{array}$ & $\begin{array}{l}\text { Estimate: } 2.14 \\
\text { p-value }<\mathbf{0 . 0 5}\end{array}$ & $\begin{array}{l}\text { Estimate: } 0.509 \\
\text { p-value: not sig. }\end{array}$ \\
\hline
\end{tabular}

3

4

5

6

7

8

9

10

11

12 


\section{Figure legends}

2 Figure 1. Average myocardial T1 relaxation times at the three different time points. Overall

3 average myocardial relaxation times followed over time; pre-surgery, 3- and 12-months post

$4 \quad$ AVR for native T1 values with 3D-QALAS and MOLLI. The p-values are from the linear

5 mixed model test and represent changes in each individual segment over the three time points.

8 Figure 2. Average myocardial T2 relaxation times at the three different time points. Overall 9 average myocardial relaxation times followed over time; pre-surgery, 3- and 12-months post 10 AVR for T2 values with 3D-QALAS and T2-GraSE. The p-values are from the linear mixed 11 model test and represent changes in each individual segment over the three time points.

Figure 3. Average percentage differences in relaxation times for each myocardial segment.

15 Blue indicates a decrease in relaxation time, while red indicates an increase between preoperative relaxation time and three respectively 12 months postoperative relaxation time.

Figure 4: Illustration of mid-ventricular maps with 3D-QALAS, MOLLI and GraSE in a patient with severe AS. The T1 and T2 relaxation times maps are acquired at three different time points: pre AVR, 3 months post AVR and 12 months post AVR. 


\section{Figures}
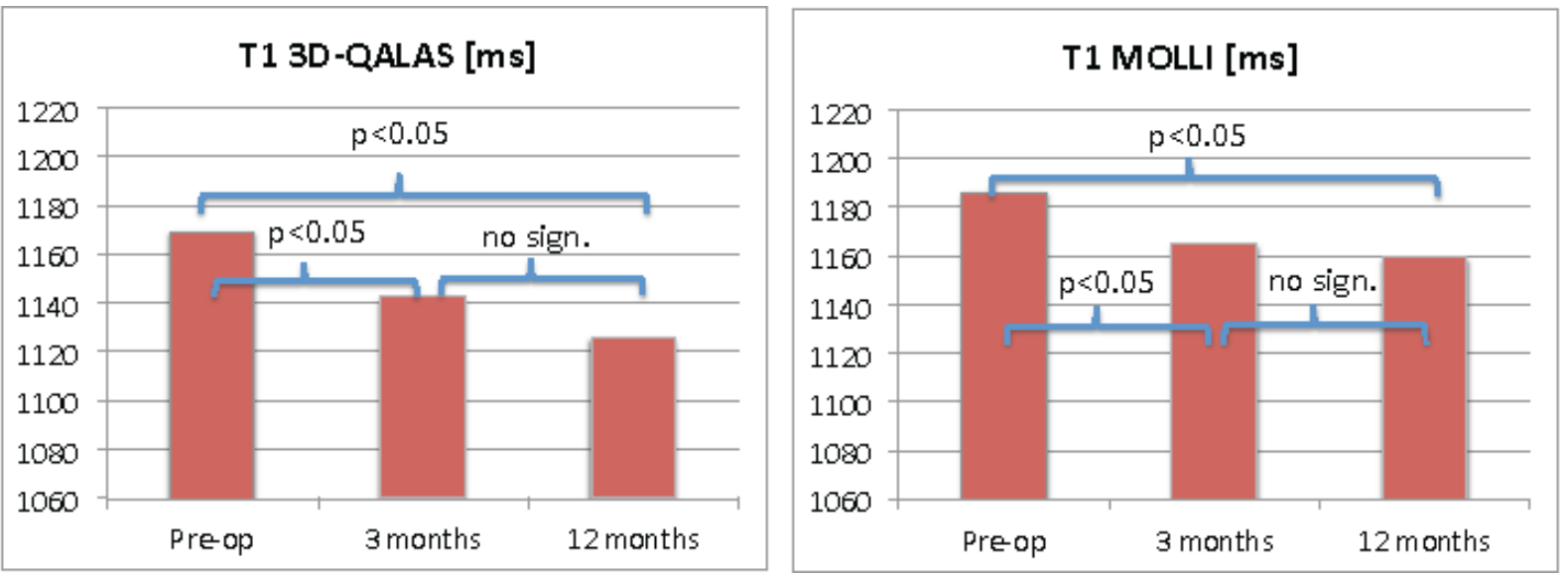

3 Figure 1. Average myocardial T1 relaxation times at the three different time points. Overall

4 average myocardial relaxation times followed over time; pre-surgery, 3- and 12-months post

5 AVR for native T1 values with 3D-QALAS and MOLLI. The p-values are from the linear

6 mixed model test and represent changes in each individual segment over the three time points.
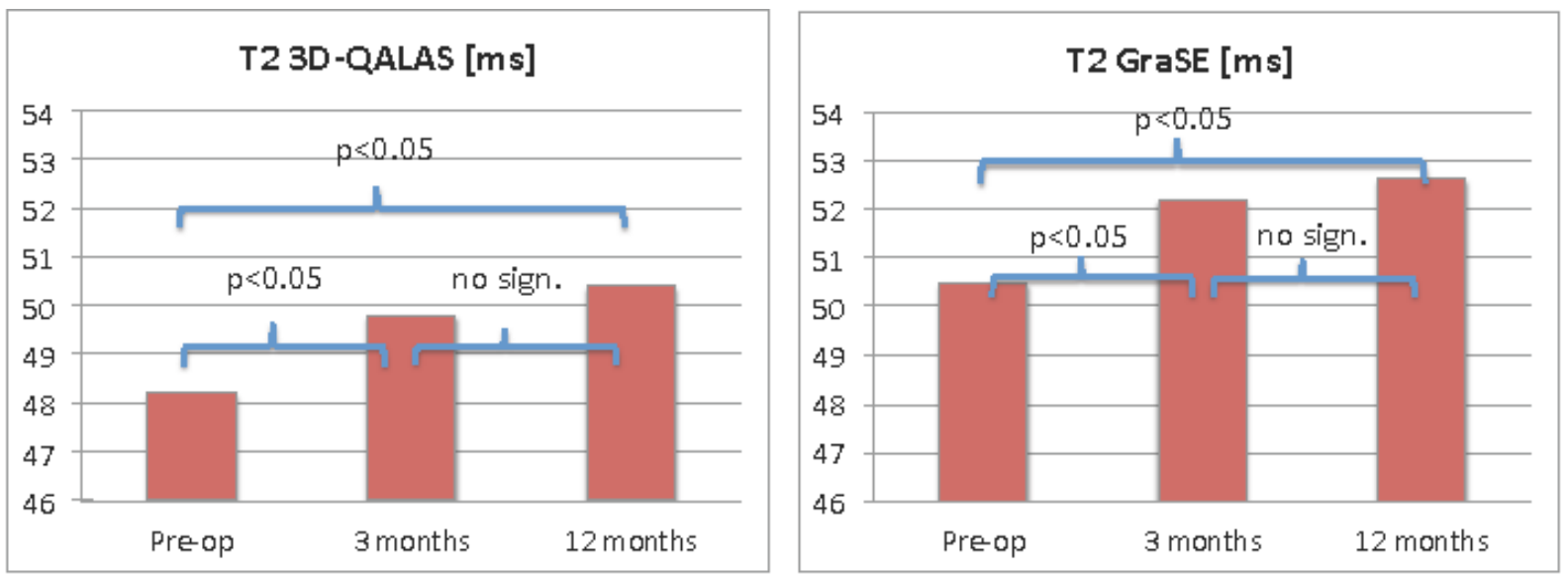

10 Figure 2. Average myocardial T2 relaxation times at the three different time points. Overall

11 average myocardial relaxation times followed over time; pre-surgery, 3- and 12-months post

12 AVR for T2 values with 3D-QALAS and T2-GraSE. The p-values are from the linear mixed

13 model test and represent changes in each individual segment over the three time points. 


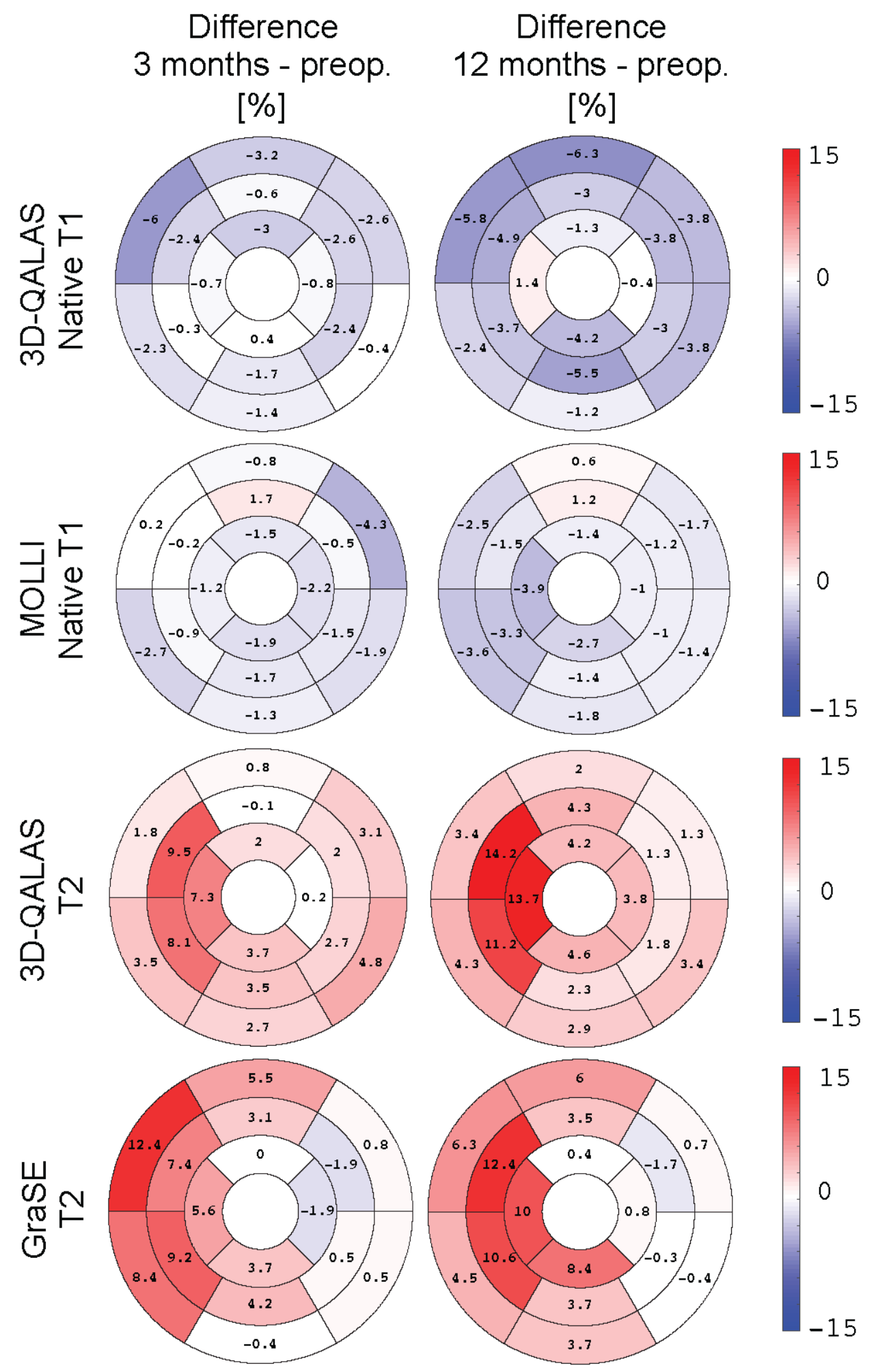

Figure 3. Average percentage differences in relaxation times for each myocardial segment.

4 Blue indicates a decrease in relaxation time, while red indicates an increase between

5 preoperative relaxation time and three respectively 12 months postoperative relaxation time. 

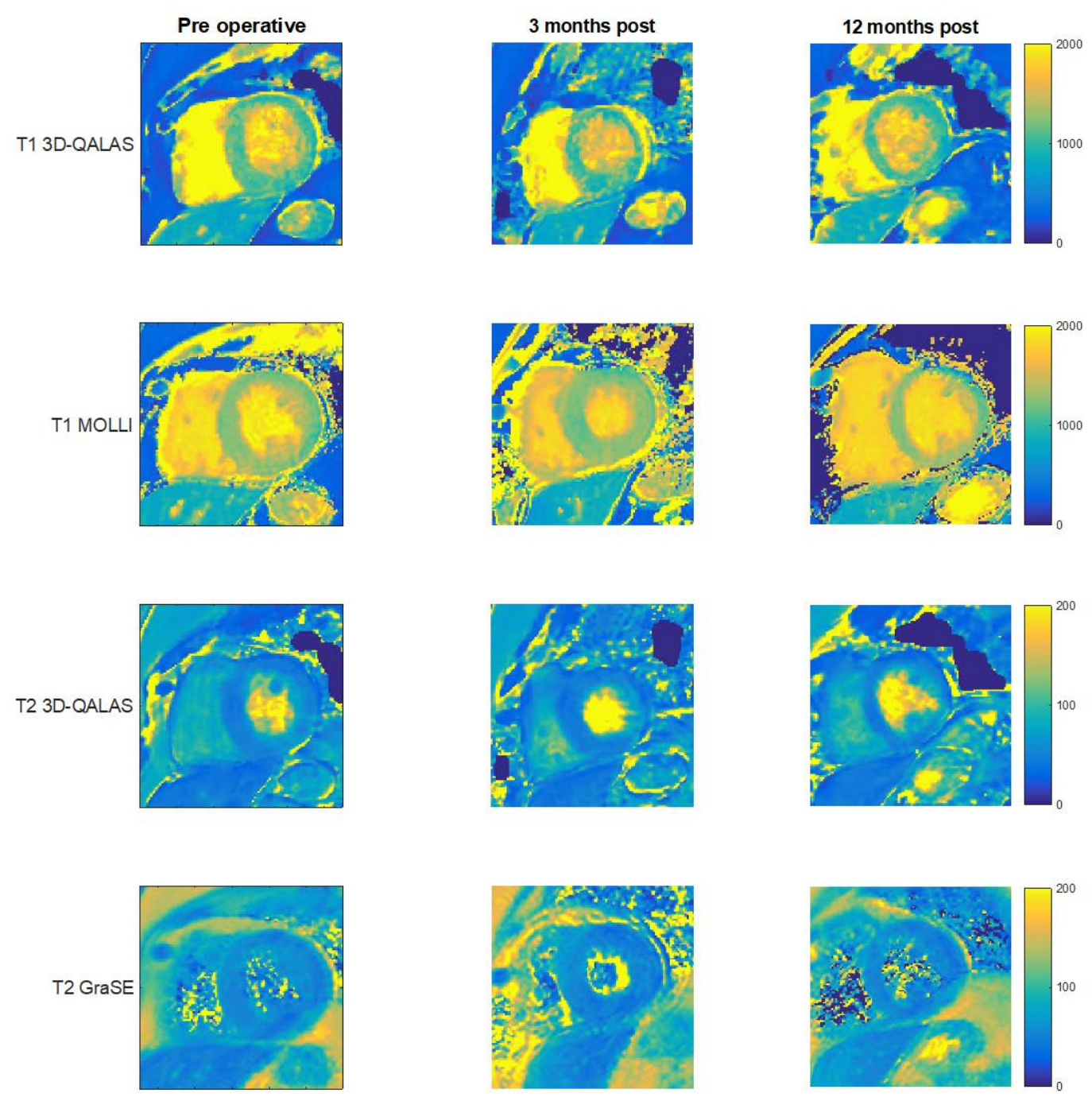

$4 \quad$ Figure 4: Illustration of mid-ventricular maps with 3D-QALAS, MOLLI and GraSE in a

5 patient with severe AS. The T1 and T2 relaxation times maps are acquired at three different

6 time points: pre AVR, 3 months post AVR and 12 months post AVR.

7 
Severe AS patients (April 2014-Dec 2015)

$\mathrm{TAVI}+$ open heart AVR

$$
n=274
$$

\section{TAVI \\ $n=139$}

Open heart AVR

$$
n=135
$$

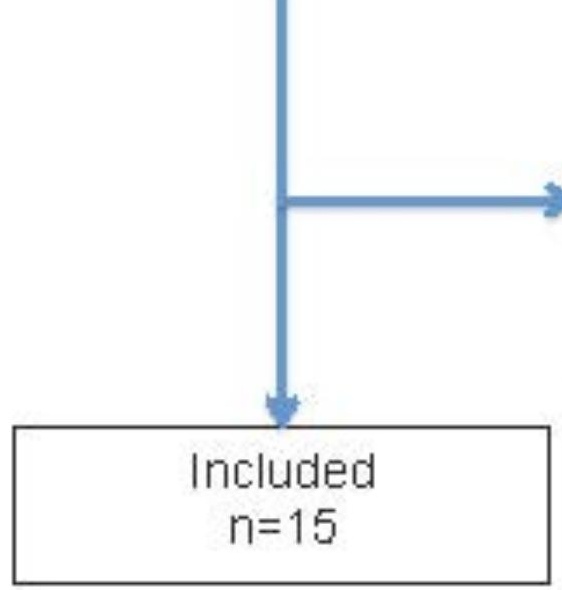

Declined to participate, pacemaker, atrial fibrillation, arrhythmia, concomitant cardiac disease, claustrophobia

$$
n=120
$$




\section{T2 3D-QALAS [ms]}

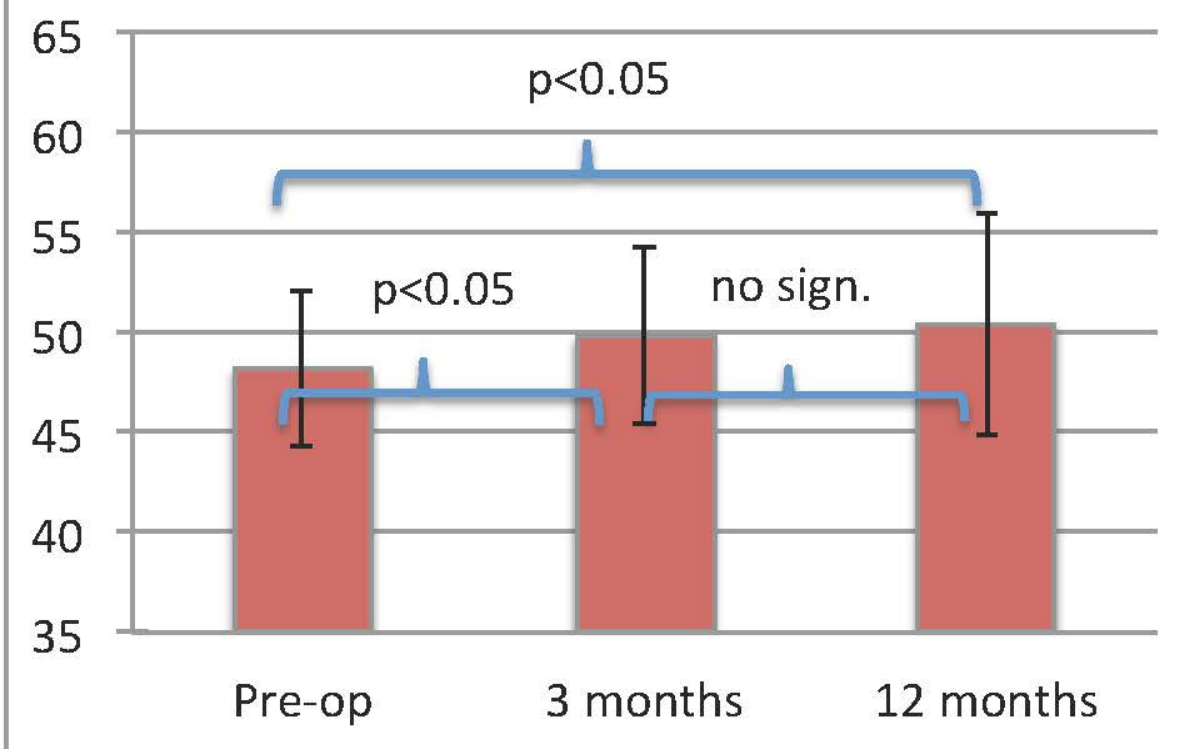

T2 GraSE [ms]

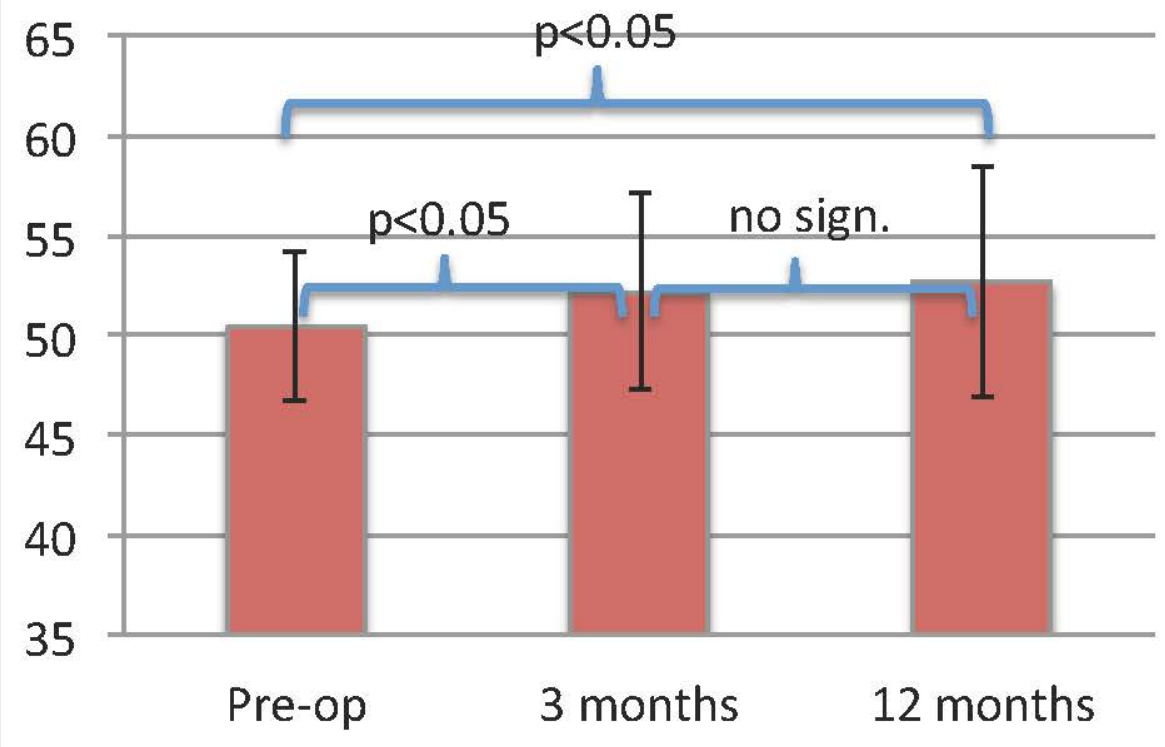




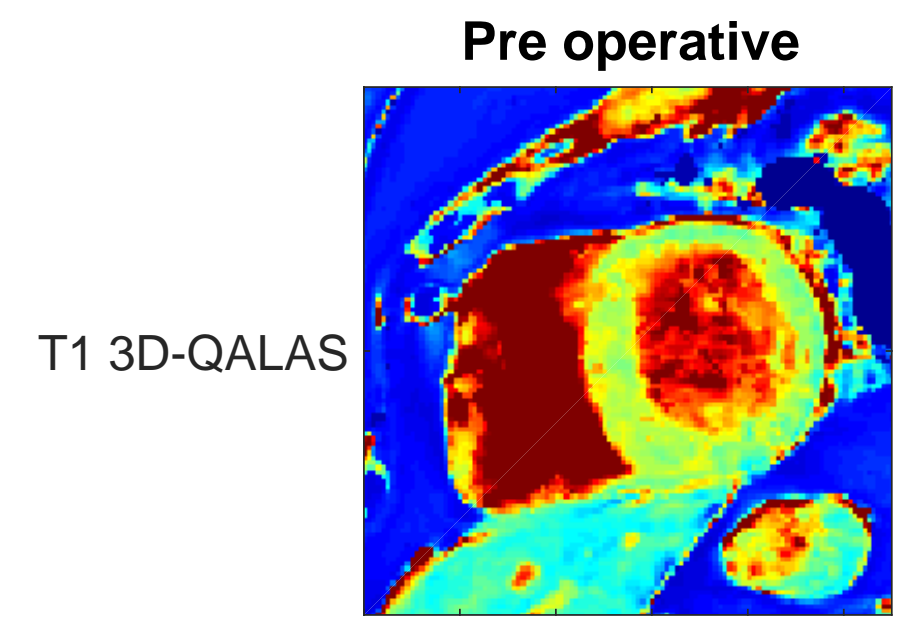

3 months post

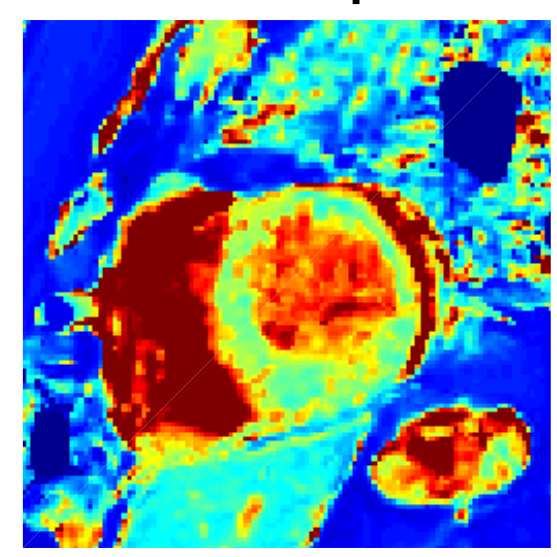

T1 MOLLI

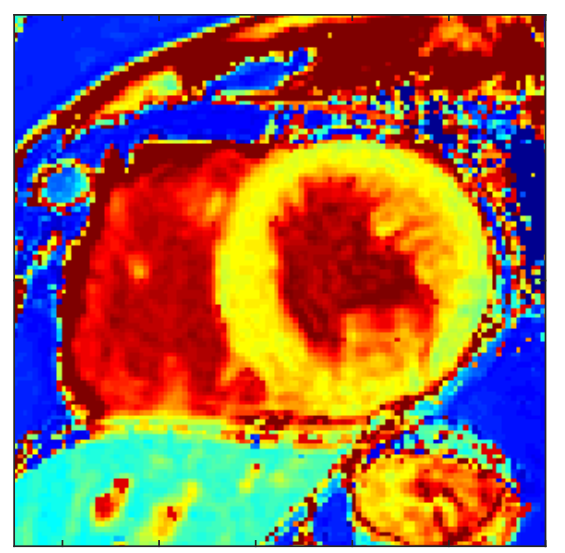

T2 3D-QALAS

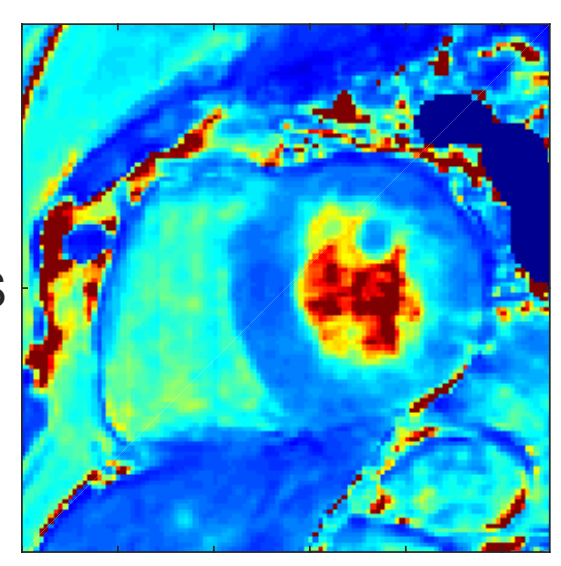

T2 GraSE

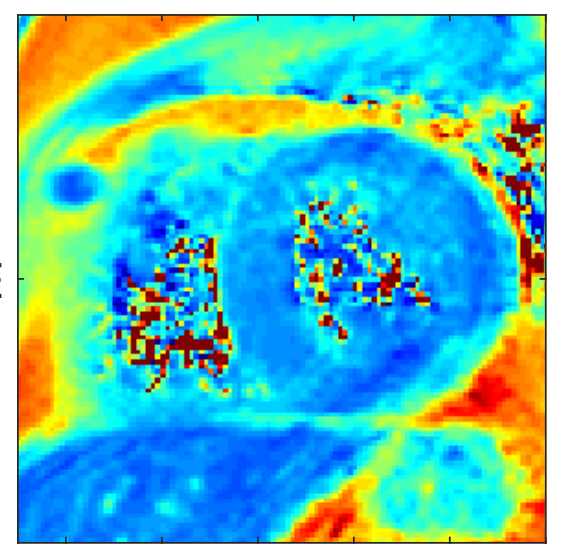

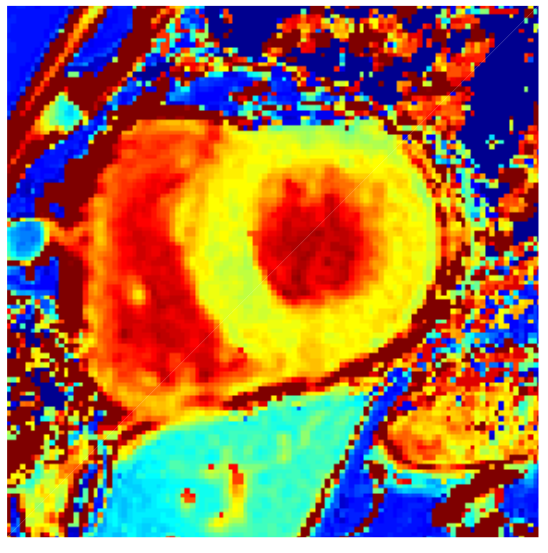
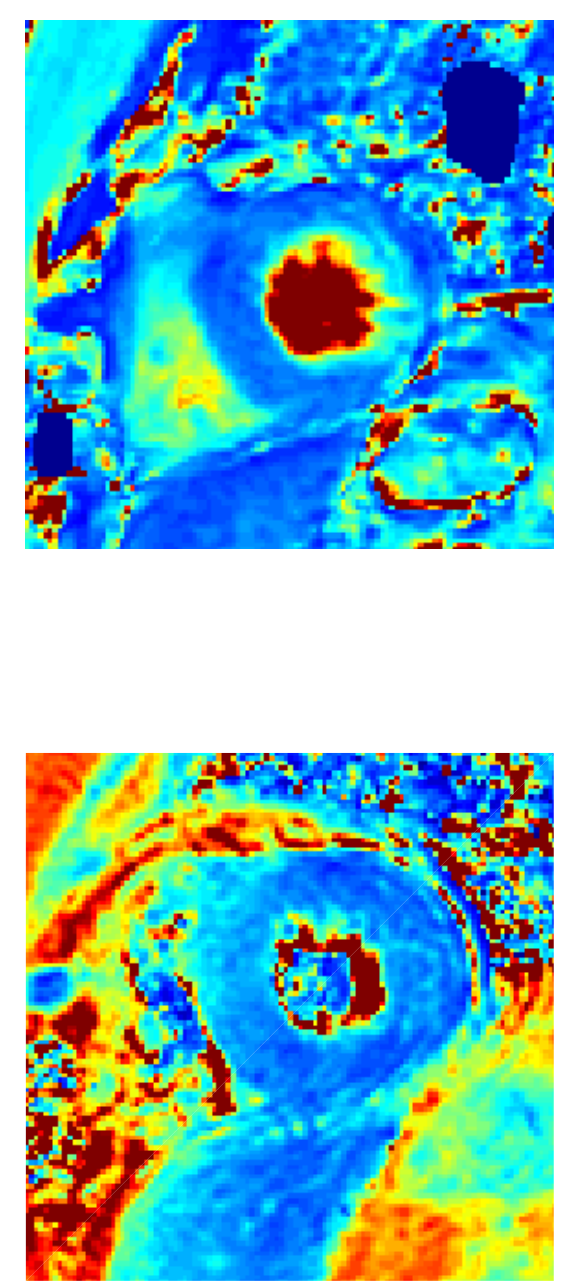

12 months post
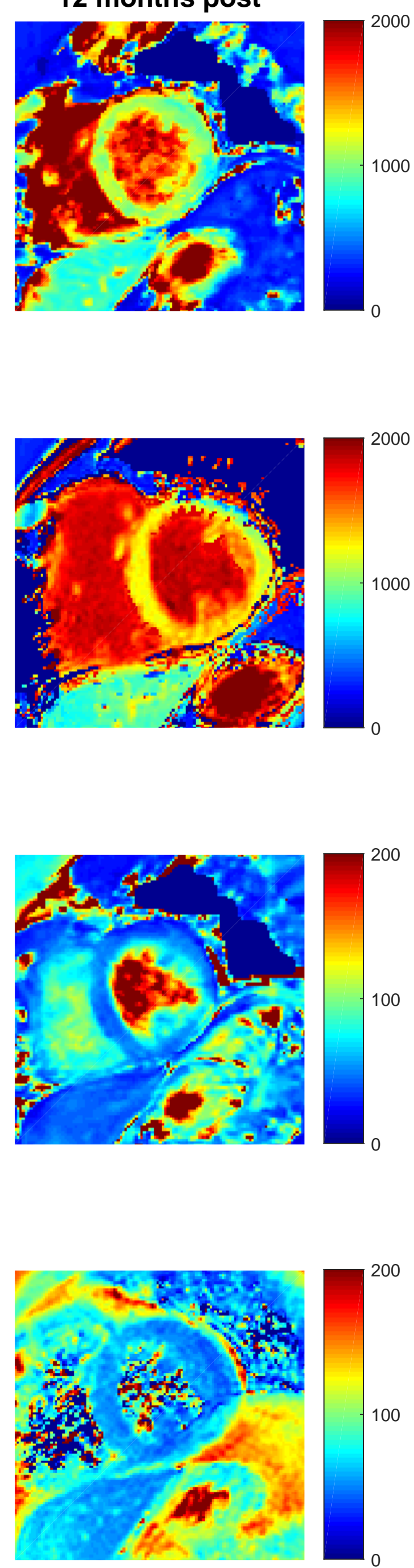